\title{
Relación entre sostenibilidad y turismo colaborativo a partir de las quejas recibidas en el alojamiento en vivienda vacacional
}

\author{
Jacques Bulchand-Gidumal \\ Santiago Melián-González \\ Universidad de Las Palmas de Gran Canaria
}

\begin{abstract}
Resumen
En este artículo se analiza la relación entre el turismo colaborativo y la sostenibilidad. Para ello se utilizan más de 6.000 valoraciones hechas por huéspedes de alquiler vacacional descargadas automáticamente de Airbnb. Sobre esta muestra se realizó un análisis de contenido con el objetivo de clasificar las principales quejas de los huéspedes. La principal conclusión es que resulta complicado establecer una relación directa entre economía colaborativa y sostenibilidad, en línea con la poca evidencia existente sobre la interacción entre los dos fenómenos. Se presentan algunas cuestiones a tener en cuenta por los distintos agentes en el desarrollo de esta nueva modalidad turística. Entre otras, la necesidad de analizar si se produce una mejor distribución de la renta turística en el territorio y el interés de los usuarios por disfrutar de un tipo de turismo distinto, en el que puedan conocer personalmente al anfitrión que oferta el alojamiento.
\end{abstract}

Palabras clave: economía colaborativa, turismo colaborativo, alojamiento colaborativo, Airbnb.

Clasificación JEL: L83, L96.

\section{Abstract}

In this manuscript, the relationship between collaborative tourism and sustainability is analyzed. To this aim, more than 6,000 reviews made by guests of this type of accommodations and downloaded automatically from Airbnb were used. A content analysis was carried out on this sample with the objective of classifying the main complaints of the guests. The main conclusion is that it is difficult to establish a direct relationship between collaborative economy and sustainability, in line with the scarce existing evidence on the interaction between these two phenomena. Some elements are presented to be taken into consideration by the different agents in the development of this new tourist modality. Among others, the need to analyze whether there is a better distribution of tourist income in the territory, and the interest of users to enjoy a different kind of tourism, in which they can personally meet the host who offers the accommodation in which they will stay.

Keywords: collaborative economy, collaborative tourism, collaborative accommodation, Airbnb.

JEL classification: L83, L96. 


\section{Introducción}

En los últimos tiempos el sector turístico se ha visto sometido a un cambio de dimensiones considerables, debido a la aparición de la que se ha dado en llamar economía colaborativa (sharing economy, en su acepción en inglés americano; collaborative economy en su acepción en inglés en Europa) y, por extensión, el denominado turismo colaborativo. Se trata de un caso en el cual los particulares prestan servicios turísticos, en principio, de forma amateur o semiprofesional, usando para ello plataformas digitales en línea y con un elevado grado de independencia a la hora de decidir qué productos o servicios ofertar, a qué precios o con qué características.

Estas plataformas se han desarrollado y popularizado en distintos subsectores dentro del turismo. Por ejemplo, Airbnb, Couchsurfing, IntercambioDeCasas y HomeAway en el caso del alojamiento, BlaBlaCar y Uber en el caso del transporte, ToursByLocals, Vayable y Airbnb Experiences en el caso de las actividades y tours, SocialCar, Drivy y Turo para el alquiler de coches entre particulares, y EatWith y VizEat para particulares que ofrecen experiencias gastronómicas en sus casas. Se trata de un sector incipiente y en desarrollo, por lo que en los próximos años es de esperar que asistamos al desarrollo de nuevas plataformas, la desaparición de muchas de las mencionadas o la consolidación de algunas de ellas, así como la aparición de plataformas que intermedien en áreas todavía al margen de las mismas.

Como ya se ha indicado, estas plataformas del denominado turismo colaborativo están empezando a suponer un profundo impacto en el sector turístico, influencia que, según las proyecciones disponibles, irá creciendo en el tiempo. Actualmente, se estima que su impacto en el sector es inferior al 1 por 100, si bien, partiendo de diversos informes publicados, no es complicado concebir un futuro en el que este tipo de servicios puedan llegar a suponer el 20 por 100 o más del total del gasto turístico si se excluye el transporte aéreo. No obstante, se requiere más evidencia sobre este tema ya que, por ejemplo, en el caso de la Comunidad Autónoma de Canarias, en 2015 , los turistas alojados en alquiler vacacional representaron el 9,6 por 100 del total, mientras que las camas ofertadas en esta modalidad supusieron el 22,3 por 100 del total de camas turísticas².

En esta situación, un tema que se plantea habitualmente es si este tipo de turismo colaborativo es un turismo más o menos sostenible que el turismo que podríamos denominar tradicional. Es decir, exclusivamente desde el punto de vista de la sostenibilidad, ¿es preferible que un turista se aloje en un hotel o es preferible que se aloje en una vivienda vacacional de un particular? En la sección sobre la revisión de la literatura se muestra la evidencia disponible sobre la relación entre el alojamiento colaborativo y la sostenibilidad. Como se comprobará, aunque la sostenibilidad de esta modalidad turística es un argumento favorable, empleado de manera frecuente por sus promotores, los datos existentes al respecto son escasos, contradictorios $y$,

\footnotetext{
${ }^{2}$ http://www.gobiernodecanarias.org/cmsgobcan/export/sites/turismo/downloads/Alquiler_vacacional/ Viviendas-Vacacionales-Canarias-2015.pdf.
} 
por tanto, inconclusos. Así, desde el punto de vista del consumidor, podemos encontrar que el coste es el motivo principal para usar el alojamiento colaborativo (Böcker y Meelen, 2017). Por otra parte, teniendo en cuenta la distribución territorial de la actividad, hay evidencia de su localización en zonas no turísticas (Gutiérrez et al., 2017), lo que conlleva una distribución más equilibrada de su impacto y renta.

En esta investigación analizamos más de 6.000 valoraciones hechas por huéspedes en Gran Canaria que han pernoctado en alojamientos contratados a través de la plataforma Airbnb. Mientras que, en la mayoría de las investigaciones similares a esta, hechas hasta la fecha, el enfoque ha sido habitualmente el de entender las causas por las que los huéspedes eligen este tipo de alojamiento, frente a los más tradicionales (hoteles y apartamentos, principalmente), en este caso analizamos las quejas principales que tienen estos clientes. El motivo de incluir en el análisis únicamente las valoraciones negativas es que, tal y como se explicará en la revisión de la literatura, en el caso del alojamiento colaborativo existen estudios que indican que, por diversas razones, muchas de las valoraciones que se realizan son excesivamente elevadas. Por ello, entendemos que las negativas, aunque sean menos en el total, pueden aportar mayor profundidad en el análisis. El objetivo es tratar de detectar en las mismas indicios acerca del tipo de turista y del tipo de valores que le caracterizan, de cara a contribuir al debate referido a la sostenibilidad del turismo colaborativo. Para este fin, utilizaremos el concepto de turismo sostenible propuesto por la Organización Mundial del Turismo que lo define como «El turismo que tiene plenamente en cuenta las repercusiones actuales y futuras, económicas, sociales y medioambientales para satisfacer las necesidades de los visitantes, de la industria, del entorno y de las comunidades anfitrionas» (Programa de las Naciones Unidas para el Medio Ambiente y Organización Mundial del Turismo, 2006, p. 12).

El presente artículo se desarrolla de la siguiente forma. En primer lugar, y en la siguiente sección, explicamos las principales contribuciones teóricas existentes alrededor del turismo colaborativo y planteamos el problema de investigación. Posteriormente, explicamos la metodología utilizada y los resultados obtenidos, finalizando con una discusión, unas conclusiones, las principales limitaciones del presente estudio y algunas sugerencias de líneas futuras de investigación.

\section{Revisión de la literatura y problema de investigación}

\subsection{Turismo colaborativo}

El turismo es una de las actividades en las que con más intensidad se ha desarrollado la economía colaborativa. Aunque esta última no es un fenómeno homogéneo, existe consenso en que comprende tres actores: el proveedor (mayoritariamente un individuo particular), la plataforma (que actúa como intermediaria) y el consumidor (que puede ser tanto un particular como una empresa u organización) (BulchandGidumal y Melián-González, 2016). Así, las dos mayores plataformas de economía 
colaborativa actualmente existentes, Airbnb y Uber, actúan en el sector turístico y representan perfectamente esta relación a tres bandas. A las mismas se pueden añadir otras modalidades menos conocidas pero que también han irrumpido en este sector, tales como ToursBylocals (experiencias turísticas) y Eatwith (experiencias gastronómicas).

Distintos autores apuntan que el imparable crecimiento de esta modalidad turística hace que aún no se conozca el fenómeno en toda su extensión y demandan más estudios, al objeto de entender correctamente sus implicaciones sobre el sector (Cheng, 2016). No obstante, sí que parece haber un cierto consenso acerca de que el turismo colaborativo ha crecido al dar respuesta, en principio, a algunas de las cuestiones que más preocupan al sector: el impacto medioambiental y el consumo de recursos, el cambio climático, la distribución de riqueza y el desarrollo sostenible (Burns y Bibbings, 2009; Dredge y Gyimóthy, 2015; Mosedale, 2012).

De forma más específica, Dredge y Gyimóthy (2015) encuentran que el turismo colaborativo permite aprovechar recursos infrautilizados así como conocimiento latente; disminuye los costes de transacción y mejora la transparencia informativa en las relaciones entre los actores; permite hacer frente al exceso de regulación en el sector, que tradicionalmente ha reducido la innovación en el mismo; y permite que el turista encuentre experiencias menos industrializadas y estandarizadas, de acuerdo con la era actual.

\subsection{Alojamiento colaborativo}

El alojamiento colaborativo puede producirse de varias formas. Tal como se explica en Bulchand-Gidumal y Melián-González (2016), las opciones se pueden clasificar en el continuo en el que se desarrolla la economía colaborativa. En un extremo estarían los intercambios en los que no hay una búsqueda de lucro. Un ejemplo de este caso sería Couchsurfing, donde los anfitriones no cobran a los huéspedes por el alojamiento. En el otro extremo, se sitúan los intercambios basados en la búsqueda del lucro. La plataforma Airbnb encaja en esta opción, ya que los anfitriones ponen tarifas a sus ofertas de alojamiento. En el medio de estos dos polos se encuentran los intercambios en los que se pretenden cubrir gastos o intercambiar de manera temporal un bien por otro. Así, en Homexchange los usuarios intercambian sus viviendas temporalmente, pudiendo este intercambio ser síncrono o asíncrono (cuando se trata de una segunda vivienda).

A pesar de la variedad anterior, es evidente que la actividad que promueven plataformas como Airbnb o Homeaway es la que más ha trascendido al público y, también, la que tiene cifras más importantes. Así, se estima que, en 2017, Airbnb oferta 4 millones de habitaciones en 191 países, cantidad que supera el número de habitaciones que tienen las cinco mayores cadenas hoteleras del mundo. En total, el número de estancias que han utilizado la plataforma como canal intermediario supera ya los 200 millones (Airbnb, 2017). 
Respecto al uso del alojamiento colaborativo, Guttentag y Smith (2017) indican que las estancias de este tipo generan experiencias locales más auténticas para los visitantes y que, en general, los usuarios encuentran mayor grado de satisfacción respecto a los hoteles, incluso en cuestiones en los que estos últimos deberían lograr mejores resultados (limpieza, por ejemplo). En cambio, encuentran que las estancias en vivienda vacacional puntúan peor que los hoteles, sea cual sea el tipo de hotel, en el check-in y el check-out, lo cual es relativamente lógico al tratarse de un proceso de puesta de acuerdo entre dos particulares, frente al caso de la recepción de hotel que habitualmente está disponible para que el huésped llegue a la hora que más le convenga.

\subsection{Sostenibilidad y turismo colaborativo}

Muy frecuentemente se ha visto a la economía colaborativa como una alternativa sostenible a la economía actual (Heinrichs, 2013; Martin, 2016). El turismo no es una excepción a esta visión.

La relación entre sostenibilidad y turismo colaborativo se inicia con el consumidor, ya que hay algunos autores que opinan que el consumo en la economía colaborativa se explica, entre otros motivos, por una mayor conciencia medioambiental (Cañigueral 2014; Hamari et al., 2015). A su vez, el menor impacto en el medioambiente es uno de los tópicos que los defensores de esta economía suelen incluir en su discurso. El razonamiento empleado es que, al promoverse un comportamiento de compartir y de acceso temporal a un bien, en contraposición al de la posesión definitiva del mismo, la producción de los bienes, con las consecuencias medioambientales que conlleva, será más racional e inferior (Sundararajan, 2014).

No obstante, y aunque en los análisis teóricos iniciales, y en las primeras investigaciones hechas con casos muy concretos, sí aparecían estas motivaciones medioambientales, recientes estudios han obtenido resultados contradictorios. Böcker y Meelen (2017) analizan por qué se usa cada uno de los ámbitos del turismo colaborativo, encontrando que en el caso del alojamiento compartido el factor principal es el económico, siendo los factores medioambientales relevantes en los casos del transporte, mientras que los factores sociales son más relevantes en el caso de las experiencias. De igual forma, Palgan et al. (2017) analizaron un grupo de usuarios, entre los cuales la sostenibilidad no fue una de las motivaciones citadas para usar el alojamiento colaborativo.

En este mismo sentido, resulta frecuente escuchar opiniones al respecto de que el turismo colaborativo, y especialmente el alojamiento colaborativo, favorece una mejor distribución del gasto en el territorio, al permitir al turista quedarse fuera de los círculos turísticos clásicos. Ello hace que se incremente el gasto en zonas tradicionalmente menos beneficiadas por dicho sector y que se involucre al residente en la actividad turística. No obstante, los estudios científicos desarrollados hasta la fecha no encuentran este tipo de comportamientos. Así, Dredge y Gyimóthy (2015) 
advierten de que, al menos hasta ahora, la expansión de una plataforma como Airbnb ha estado muy centrada en ciudades y en zonas muy concretas de las mismas, dejando casi completamente de lado el medio rural y las zonas menos turísticas de las ciudades. No obstante, indican la necesidad de llevar a cabo más investigación en este sentido. Por su parte, un reciente artículo publicado por Gutiérrez et al. (2017) encuentra patrones espaciales que apoyan ambos puntos de vista. Por un lado, detectan una mayor concentración de alojamientos en Airbnb que de hoteles en zonas centrales y cerca de las atracciones turísticas, generando una mayor presión urbanística y turística en dichas áreas; pero, por otro lado, también encuentran alojamientos ofertados en Airbnb en zonas residenciales en las cuales no hay hoteles, es decir, en zonas que hasta ahora habían quedado ajenas al circuito turístico.

También incluimos como parte de la relación entre sostenibilidad y turismo colaborativo el efecto de este en la duración de la estancia y el gasto total. De nuevo en este caso, los resultados no son definitivos, pero van indicando algunos patrones. En primer lugar, parece confirmarse que los turistas que se hospedan en viviendas vacacionales realizan estancias más largas (Fang et al., 2016; Guttentag y Smith, 2017; Tussyadiah y Pesonen, 2016), realizan mayor número de viajes a lo largo del año y un mayor número de actividades durante los viajes (Tussyadiah y Pesonen, 2016). Todo ello genera empleo en el sector, al expandirse el mismo, a la vez que también se producen nuevas oportunidades. No obstante, y dado que parece que parte de estas estancias en viviendas vacacionales causan la disminución de estancias en los hoteles más económicos de la zona (Zervas et al., 2017), se producirá un efecto contrario al anterior, al reducirse el empleo en este tipo de hoteles. Este efecto se verá, además, acompañado del menor gasto por parte del turista alojado en vivienda vacacional frente al alojado en hotel (Guttentag y Smith, 2017). Es decir, que tal y como explican Fang et al. (2016) el efecto marginal de creación de empleo puede ser menor a medida que aumenta el tamaño de alojamiento colaborativo.

Por último, Palgan et al. (2017), y desde el punto de vista estricto de la sostenibilidad medioambiental, hablan de un posible efecto rebote: al aumentar el tiempo total de estancia y el número de estancias gracias al turismo colaborativo, se puede producir un efecto de mayor consumo total, al generar mayor número de desplazamientos, consumo de recursos naturales, etc. En este sentido, un estudio realizado por la propia Airbnb (2014) indica que las estancias en alojamientos contratados a través de su web generan un menor consumo de agua y de energía, menos emisión de gases de efecto invernadero y menor cantidad de residuos. No obstante, se trata de un estudio realizado en 2014, cuando el fenómeno estaba aún en evolución, y por la propia plataforma, por lo que entendemos que los resultados habría que tomarlos con una cierta cautela. Aun así, entendemos que puede ser una línea interesante a investigar en el futuro. 


\subsection{Problema de investigación}

Como ya se ha comentado anteriormente, los artículos disponibles hasta el momento en la literatura sobre la economía colaborativa parecen agruparse en tres tópicos principales.

En primer lugar, las razones por las cuales los clientes utilizan este tipo de servicios (Hamari et al., 2015), mencionándose la sostenibilidad, razones económicas o razones de conveniencia de los servicios ofrecidos, entre otras muchas. En este ámbito, hay pocos artículos que traten específicamente el caso del alojamiento colaborativo.

En segundo lugar, hay artículos que analizan el impacto de la economía colaborativa en un sector específico (Zervas et al., 2017), los problemas globales de la economía colaborativa (Malhotra y Van Alstyne, 2014) e, incluso, en artículos recientes, se ha empezado a trabajar ya el concepto de cómo atraer más reservas en el ámbito de la economía colaborativa, al analizar el proceso de compra que llegan a cabo los consumidores (Ert et al., 2016). En este bloque, sí encontramos algunos artículos que tratan el ámbito del alojamiento colaborativo, casi siempre usando el caso de Airbnb.

Finalmente, otra línea clara de publicaciones se centra en torno al tipo de trabajo que se está generando por el desarrollo de esta economía (Codagnone et al., 2016). Esta línea de investigación también está bastante alejada del alojamiento colaborativo.

En cambio, una perspectiva que ha recibido poca atención hasta el momento es la relativa a la satisfacción de los clientes con los servicios de la economía colaborativa y en qué medida este tipo de consumidores pueden hacer más sostenible el desarrollo turístico del destino. Por ahora, uno de los pocos estudios disponibles se centra en el hecho de lo positivas que son las evaluaciones online en sitios como Airbnb (Zervas et al., 2015).

En este sentido, hay que tener en cuenta que cualquier estudio que trate de analizar la perspectiva del cliente en la economía colaborativa debe tener en cuenta algunas cuestiones. Primero que, por ahora, el porcentaje de población total que ha usado los servicios de la economía colaborativa es relativamente bajo y, por ello, el proceso de selección de una muestra representativa es complejo.

Segundo, si se analizan las evaluaciones de una forma tan solo cuantitativa se corre el riesgo de que los datos obtenidos tengan escaso valor, ya que, tal y como demuestran algunos autores (por ejemplo, los ya mencionados Zervas et al., 2015), en general las valoraciones suelen ser positivas, con una fuerte restricción en el rango de las puntuaciones. La explicación de esta característica puede residir en el contacto directo entre consumidor y proveedor, los acuerdos a los que pueden llegar los mismos al margen de la plataforma, y el posible miedo a la venganza, dado que no solo el huésped valora al anfitrión, sino también el anfitrión al huésped. No obstante, este último problema ha quedado en principio resuelto al no publicar las plataformas estas valoraciones hasta haber recibido ambas, evitando el posible efecto mencionado. 
Tercero, en la mayoría de los casos no es posible contactar directamente con los usuarios de estas plataformas, ya que las mismas suelen ocultar sus datos de contacto. Además del intento de proteger la privacidad de los consumidores, entendemos que esta situación también puede deberse a que las plataformas tratan de dificultar el contacto directo entre los proveedores de servicios y los clientes, ya que en caso de conseguirlo podrían evitar a la plataforma y, por ello, sus comisiones.

Por todo ello, definimos el problema de investigación de este artículo como analizar, a través de los comentarios de consumidores insatisfechos, si el alojamiento colaborativo puede incidir positivamente en la sostenibilidad del destino en el que opera. La evidencia en cuanto a esto puede provenir de opiniones directamente relacionadas con la sostenibilidad de la experiencia de alojamiento y de los hábitos que se pueden inferir de las mismas.

\section{Metodología}

De cara a evitar los problemas citados en el apartado anterior, principalmente el relativo al hecho de lo elevadas que son las valoraciones en el caso del alojamiento colaborativo, y tratando de obtener una perspectiva real de lo que opinan los clientes del alojamiento colaborativo y de su relación con la sostenibilidad, en esta investigación se llevó a cabo una descarga masiva de datos del principal portal de alojamiento colaborativo, Airbnb.

En concreto, se descargaron todas las opiniones recibidas, a través de Airbnb, por todas las propiedades existentes en un territorio geográfico determinado (Gran Canaria). Esta descarga ha sido factible debido a que, en la actualidad, la API de acceso a los datos de Airbnb está disponible para todo aquel que la quiera utilizar.

Así, se descargaron un total de 10.755 evaluaciones de clientes. Para cada una de ellas está disponible la fecha en que se realizó la misma, los comentarios realizados por el viajero, el idioma empleado en la evaluación y el alojamiento específico para el cual se hizo la valoración. Adicionalmente, para cada uno de estos alojamientos están disponibles las características del mismo: precio medio, tipo de vivienda del que se trata, tipo de alquiler que se ofrece y número de valoraciones que ha recibido. De todas ellas, se analizaron aquellas que estaban en español o inglés, que representan el 56,2 por 100 del total (6.040 opiniones).

De cara al análisis de contenido, se llevaron a cabo varios procedimientos. En primer lugar, una investigadora del equipo de investigación al que pertenecen los autores leyó cada una de las evaluaciones y las clasificó en uno de los siguientes cinco grupos: a) sin mención a ningún aspecto negativo; b) positiva, pero con algún punto muy concreto a mejorar; c) con aspectos positivos y negativos por igual; d) negativa, pero con aspectos positivos; y e) solo negativa, sin mención a aspectos positivos. A la vez, para cada evaluación que tenía uno o más comentarios desfavorables se transcribieron los mismos de manera literal. Posteriormente, los dos investigadores principales del proyecto revisaron esta clasificación y extracción de textos literales para asegurar su correcta categorización y desarrollo posterior. 
Una vez disponible el porcentaje de opiniones en las que no aparece ningún elemento negativo reflejado (4.938 de las opiniones, que corresponden al 81,8 por 100 del total) se llevó a cabo un análisis de contenido de las valoraciones transcritas que contenían uno o más comentarios desfavorables (es decir, aquellas pertenecientes a los cuatro últimos de los cinco grupos descritos en el párrafo anterior). Así, los dos autores de este trabajo, de forma independiente y a partir de la clasificación otorgada a las opiniones con algún comentario negativo, analizaron el contenido desfavorable transcrito. De esta manera, en base al significado de las opiniones negativas, se generaron dos agrupaciones independientes de causas de insatisfacción de los clientes. El grado de acuerdo entre las dos agrupaciones fue superior al 97 por 100. Las diferencias se resolvieron mediante consenso y se logró una clasificación definitiva. También se cruzaron estas cuestiones mencionadas con variables como el precio y el tipo de alquiler que se llevaba a cabo (casa completa, habitación privada, habitación a compartir).

\section{Resultados}

En primer lugar, presentamos una breve clasificación de las estancias realizadas. Como ya se indicó, en total se analizaron 6.040 opiniones, clasificadas según distintos parámetros tal y como se muestra en el Cuadro 1. En la misma se puede observar que la mayoría de la muestra (75 por 100 del total) está referida a viviendas alquiladas en su totalidad, siendo casi marginal los casos de habitaciones compartidas. Los precios medios por noche en más del 90 por 100 de los casos son inferiores a 100 euros por noche, siendo el tipo de propiedad más habitual el apartamento (70 por 100) y las casas (20 por 100). En cuanto a si las propiedades aceptan reservas instantáneas, el 49 por 100 lo permite y el 51 por 100, no.

En cuanto al análisis realizado, el primer resultado es que, tal y como ya se ha indicado, en general, la gran mayoría de las opiniones encontradas no tienen ningún tipo de comentario negativo o desfavorable $(81,8$ por 100$)$. A tener en cuenta que, aunque algunas de las mismas no parecen indicar un grado de satisfacción muy elevado (por ejemplo, «Casa cómoda y bien situada. El propietario amable»), en estas no se menciona ningún punto a mejorar o negativo como para poder extraer ulteriores conclusiones. Es decir, aunque no sería correcto atribuirles un nivel de satisfacción excepcional, la realidad es que en las mismas no se indica ningún elemento susceptible de mejora y, en general, la mayoría de las opiniones de este tipo analizadas, mostraban un razonable grado de satisfacción con la estancia.

Las críticas negativas encontradas fueron clasificadas en 11 categorías: tamaño de la vivienda o de alguna de sus dependencias; estructura de la vivienda; carencias de equipamiento; vivienda o equipamiento con desperfectos; limpieza; camas; incidencias durante la estancia (fueran o no resueltas las mismas durante la duración de la estancia); cuestiones relativas al edificio en el que se integra la vivienda (por ejemplo, vecinos, ruidos, ascensor, etc.); cuestiones relativas al destino o al entorno urbano en el que se encuentra la vivienda; problemas con el anfitrión (por ejemplo, 


CUADRO 1
CARACTERÍSTICAS DE LA MUESTRA ANALIZADA
\begin{tabular}{|l|r|}
\hline \multicolumn{2}{|c|}{ (En \%) } \\
Tipo de alojamiento \\
Vivienda completa & 75,1 \\
Habitación privada & 24,5 \\
Habitación compartida & 0,4 \\
\hline Precio medio por noche & \\
Hasta 50 euros & 46,2 \\
Entre 51 y 100 euros & 7,5 \\
Entre 101 y 150 euros & 4,0 \\
Más de 151 euros & 2,4 \\
\hline Tipo de propiedad & 69,6 \\
Apartamento & 19,4 \\
Casa & 4,4 \\
B\&B & 2,1 \\
Loft & 4,5 \\
Otros (cabaña, villa, barco, etc.) & 49,0 \\
\hline Aceptan reserva instantánea & 51,0 \\
\hline Sí
\end{tabular}

FUENTE: Elaboración propia.

falta de comunicación, impuntualidad, incumplimientos); y otro tipo de inconvenientes diferentes.

A continuación, se mencionan los aspectos que más frecuentemente aparecieron. Es necesario aclarar que cada una de las valoraciones analizadas puede incluir críticas relativas a más de una de las 11 categorías citadas anteriormente, por lo que la suma total es superior a 100 por 100 .

- Cuestiones relativas al destino o al entorno urbano en el que se encuentra la vivienda (45 por 100 de los casos en los que hay quejas). Se incluyen aquí el ruido en la calle que se oye desde el alojamiento, las dificultades de aparcamiento en la zona y el clima en el destino los días de la estancia.

- Carencias de equipamiento en la vivienda (25 por 100), mencionándose principalmente problemas en cuanto a escasez de equipamiento en la cocina y en el baño.

- Problemas con el anfitrión (19 por 100), siendo las más citadas cuestiones relativas a no haber tratado directamente con el mismo, las dificultades de comunicación por barreras idiomáticas y comportamientos poco adecuados del mismo.

- Tamaño de la vivienda o de alguna de sus dependencias (15 por 100), entre las cuales se cita principalmente el baño. 
- Vivienda o equipamiento con desperfectos (13 por 100). Desde luces o persianas que no funcionan hasta quejas respecto al aire acondicionado y la poca presión del agua.

Para entender mejor estas críticas, también se realizó un cruce de las mismas con variables del alojamiento, principalmente con el tipo de alquiler del que se trataba. En este caso, los siguientes fueron los principales resultados obtenidos:

- En las cuestiones relativas al destino o al entorno urbano en el que se encuentra la vivienda, no se encontraron diferencias significativas entre el tipo de alquiler del que se tratara (vivienda completa, habitación privada o habitación compartida).

- En el caso de las quejas en referencia a carencias de equipamiento en la vivienda y en aquellas referidas al tamaño de la vivienda, estas se dan con mayor frecuencia en los casos de alquiler de la vivienda completa, siendo esta diferencia significativa.

- En cuanto a las quejas que hacen referencia al anfitrión, estas se dan con mucha mayor frecuencia en los casos de alquiler de una habitación. Recordar que en el conjunto de datos analizados casi no hay casos de habitación compartida (tan solo el 0,4 por 100 de las estancias); es decir, casi todos los casos de alquiler de habitaciones hacen referencia a habitaciones privadas.

\section{Discusión}

La primera implicación del presente estudio es que, en general, hay un alto grado de satisfacción de los clientes con los servicios recibidos con el alojamiento turístico a través de Airbnb, confirmando lo encontrado en estudios previos de corte cuantitativo (Fradkin et al., 2015). Del análisis hecho en el presente estudio no se puede confirmar si estas valoraciones están infladas, indicando las mismas un valor superior al de la experiencia realmente recibida (Ert et al., 2016; Zervas et al., 2015). No obstante, dado que el resultado encontrado en esta investigación se basa en un análisis del contenido de las opiniones de los clientes, el mismo complementa y refuerza los hallazgos ya existentes en la literatura. Así, a pesar de haber hecho un análisis de contenido de las opiniones (en contraposición a tener en cuenta solo la puntuación otorgada), tan solo se han encontrado un 18 por 100 de opiniones con algún tipo de queja respecto a la estancia. Es decir, en el 82 por 100 de los casos no se menciona ninguna cuestión a mejorar, lo cual parece contradecir la situación de que las valoraciones estén infladas. Así, entendemos que si hubiera valoraciones infladas podría ser frecuente encontrar casos de una alta valoración numérica, pero que posteriormente en el comentario se indicaran algunas cuestiones negativas del alojamiento. De esta manera, el bajo nivel de quejas encontrado hace pensar que no se está dando este caso.

No obstante, esto también puede indicar la necesidad de complementar estos resultados con una investigación más directa con los clientes para tratar de entender 
si lo que se está manifestando públicamente en la plataforma es un reflejo real de su estancia. Hay que recordar que el análisis de contenido realizado ha estado enfocado a la búsqueda de quejas específicas y que, tal y como se explicó, hay opiniones que, sin ser realmente positivas, no expresan ninguna crítica en concreto y, por lo tanto, forman parte del citado 82 por 100.

La segunda implicación del presente estudio es que las críticas principales hacia los servicios de alojamiento en la economía colaborativa inciden precisamente en cuestiones que diferencian a la misma de servicios más tradicionales como el caso hotelero: relaciones con los vecinos, problemas de aparcamiento, relación con el anfitrión, tamaño de la vivienda, equipamiento disponible o tamaño de la vivienda. Muchas de estas cuestiones están resueltas en el ámbito tradicional, debido a la estandarización que se ha llevado a cabo en la industria hotelera y a la existencia de parámetros objetivos respecto a cuestiones como puede ser el tamaño mínimo de la habitación en función del número de estrellas del hotel. No obstante, esta estandarización hotelera ya ha sido reflejada en la literatura como uno de las principales paradojas que debe afrontar la misma (Sandoff, 2005) y, probablemente, sea una de las causas por las que existe un mayor interés por los nuevos modos de alojamiento turístico existentes.

La tercera implicación es que no hemos encontrado mención alguna, entre las quejas de los consumidores, a la mayor sostenibilidad del alojamiento colaborativo. Es más, determinados comentarios referidos al aire acondicionado, a la cantidad de agua caliente disponible o a la insuficiente presión del agua podrían, incluso, ser calificados como propios de huéspedes poco preocupados por el medioambiente, más teniendo en cuenta que el destino analizado no se caracteriza, normalmente, por temperaturas extremas, por lo que no está generalizado en el mismo el uso del aire acondicionado, al menos entre los residentes.

Para finalizar, no es posible dejar de lado el hecho de que, globalmente, y con los datos disponibles hasta el momento, la valoración online de los alojamientos en opciones ligadas a la economía colaborativa es muy superior a la que reciben los hoteles. Aunque hay que recalcar en que hay que tener en cuenta la existencia de potenciales factores mediadores que podrían tener una incidencia muy importante en dicha valoración, no deja de ser cierto que, en general, la valoración es muy alta.

\subsection{El turismo colaborativo y la sostenibilidad}

Analizando de forma más detallada la relación entre el turismo colaborativo y la sostenibilidad, de los datos resultantes se pueden extraer algunas conclusiones preliminares. En primer lugar, el perfil del usuario de la economía colaborativa parece inclinarse más hacia la vivienda vacacional completa (75 por 100) que al alquiler de habitaciones, sean estas privadas o compartidas (25 por 100). Estos datos contradicen, en parte, la afirmación de que en la economía colaborativa hay sobre todo una oferta de recursos infrautilizados. Es cierto que un propietario puede tener una 
vivienda vacía que destina al alquiler vacacional, pero un aprovechamiento más eficiente y la mejora de la sostenibilidad entendemos que se podría dar en el caso de las habitaciones que comparten los anfitriones.

En segundo lugar, creemos que es interesante observar que las principales quejas de aquellos que se alojan en una vivienda vacacional se refieren a aspectos no directamente relacionados con la vivienda, sino con el entorno. Explicábamos al principio del presente artículo cómo, en una situación de desplazamiento de la demanda de una zona a otra de la ciudad, la economía colaborativa permitía que rápidamente hubiera a disposición de los turistas una oferta de alojamiento, sin necesidad de desarrollar grandes infraestructuras, reformas de edificios para destinarlos a hoteles, etc. Siendo esta cuestión cierta, también observamos cómo los turistas acaban, en muchos casos, echando de menos cuestiones habitualmente resueltas en un hotel, como el aparcamiento o la insonorización. Es relativamente sencillo encontrar en los medios de comunicación quejas similares en grandes ciudades europeas en las cuales determinadas zonas se han puesto de moda, generándose una oferta de alojamiento muy amplia en la zona que, por cuestión de tiempo, no ha venido respaldada del aumento de servicios públicos necesarios (por ejemplo, recogida de basuras o tipología de comercios). A nuestro juicio, es una cuestión interesante a tener en cuenta en el debate acerca de la relación entre la economía colaborativa y la sostenibilidad.

Por último, mencionar que en las quejas referidas al equipamiento de la vivienda (citadas en un 25 por 100 de las valoraciones en las que hay algún elemento negativo), la gran mayoría hacen referencia a la cocina y al baño. Igualmente, y aunque no es objeto del presente análisis, también hemos detectado en las opiniones sin elementos negativos que en muchos casos se cita como cuestión positiva la cocina del alojamiento (por su tamaño, equipamiento, etc.). Entendemos que es un tema relevante a tener en cuenta y que confirma que, como es lógico, muchos de los que alquilan vivienda vacacional lo hacen con el objetivo de disponer de independencia a la hora de comer. Esto tiene un doble efecto: por un lado, el gasto total del turista en manutención durante la estancia será menor, pero, al mismo tiempo, también se producirá un doble efecto de compra en el comercio de proximidad y de acudir a restaurantes que probablemente se encuentren fuera del circuito turístico clásico, pudiéndose dar, en su caso, una mayor distribución de las rentas generadas por el turismo.

\section{Conclusiones}

En este artículo pretendíamos analizar la relación entre el turismo colaborativo y la sostenibilidad, analizando en qué medida se trata de un turismo que puede ser más sostenible para el destino en el que se produce. A partir de un análisis de contenido de una muestra amplia de 6.040 valoraciones, realizadas por los huéspedes en el portal Airbnb, la principal conclusión es que, si bien los datos no permiten ser concluyentes, no parece ser que se den efectos positivos de la economía colaborativa hacia la sostenibilidad de los destinos turísticos. De igual forma, hay algunos 
indicios que lo que sí se puede estar generando es una mejor distribución en el territorio del gasto turístico. De forma paralela, este análisis ha permitido confirmar, por un lado, la elevada satisfacción general de los huéspedes de la economía colaborativa. Por otro lado, las principales quejas se refieren a cuestiones no relacionadas con la vivienda, al equipamiento de la misma y a cuestiones relativas a la relación con el anfitrión, destacando el no haberlo conocido personalmente.

\subsection{Limitaciones y futuras líneas de investigación}

Como ya se ha indicado, en este estudio tan solo se analizó un territorio (la isla de Gran Canaria) por cuestión de tiempo y recursos, pero puede extenderse el análisis a otras zonas geográficas permitiendo incluso la comparativa entre ellas. En segundo lugar, tan solo se analizaron las evaluaciones en español y en inglés, si bien estos dos idiomas representaban casi el 60 por 100 del total. No obstante, un análisis de evaluaciones en otros idiomas permitiría llevar a cabo comparativas entre los orígenes de los viajeros, ya que está demostrado que las exigencias, necesidades e interés por la sostenibilidad de los viajeros son distintas según su nacionalidad. En tercer lugar, no se analizaron las características de los viajeros, como si son usuarios frecuentes de la economía colaborativa, si son viajeros asiduos, si usan habitualmente otros tipos de alojamiento aparte de los disponibles en la economía colaborativa, etc. El motivo es no estar disponible este dato, por lo que no es posible conocer el detalle del perfil del viajero que puntúa en Airbnb más allá de las evaluaciones que haya hecho en dicha plataforma. En cuarto lugar, tampoco se realizaron análisis detallados al respecto de las críticas y quejas recibidas por tipo de propiedad (casa, apartamento, alojamiento y desayuno, etc.), por localización de la vivienda (zona urbana central, zona urbana periférica, pueblo, zona rural, etc.) ni por tamaño de la vivienda.

Por último, en futuras investigaciones, además de superar las anteriores limitaciones, creemos que sería interesante realizar análisis similares en otras webs de economía colaborativa como las mencionadas en la introducción (Uber, BlaBlaCar, SocialCar, EatWith), para entender mejor el perfil del turista que viaja usando el turismo colaborativo.

\section{Referencias bibliográficas}

[1] AIRBNB (2014). A greener way to travel: The environmental impacts of home sharing. Disponible en http://blog.atairbnb.com/environmental-impacts-of-home-sharing/ (visitado el 31 de julio de 2017).

[2] AIRBNB (2017). Airbnb Fast Facts. Disponible en https://press.atairbnb.com/app/ uploads/2017/08/4-Million-Listings-Announcement-1.pdf (visitado el 15 de septiembre de 2017). 
[3] BÖCKER, L. y MEELEN, T. (2017). «Sharing for people, planet or profit? Analysing motivations for intended sharing economy participation». Environmental Innovation and Societal Transitions, 23 (2017), 28-39.

[4] BULCHAND-GIDUMAL, J. y MELIÁN-GONZÁLEZ, S. (2016). Una guía para entender la economía colaborativa: de clientes-consumidores a individuos-proveedores. Autoeditado por los autores en Amazon. Disponible en https://www.amazon.es/entendereconom\%C3\%ADa-colaborativa-clientes-consumidores-individuos-proveedoresebook/dp/B01MRNC50W (visitado el 31 de julio de 2017).

[5] BURNS, P. M. y BIBBINGS, L. J. (2009). «The end of tourism?». Climate Change and Societal Changes (February 2015), 37-41.

[6] CAÑIGUERAL, A. (2014). Vivir mejor con menos. Descubre las ventajas de la nueva economía colaborativa. Barcelona: Penguin Random House Grupo Editorial.

[7] CHENG, M. (2016). «Current sharing economy media discourse in tourism». Annals of Tourism Research, 60, 111-114.

[8] CODAGNONE, C.; ABADIE, F. y BIAGI, F. (2016). «The Future of Work in the 'Sharing Economy'. Market Efficiency and Equitable Opportunities or Unfair Precarisation?». Institute for Prospective Technological Studies, JRC Science for Policy Report EUR 27913 EN, doi:10.2791/431485.

[9] DREDGE, D. y GYIMÓTHY, S. (2015). «The collaborative economy and tourism: Critical perspectives, questionable claims and silenced voices». Tourism Recreation Research, 40 (3), 286-302.

[10] ERT, E.; FLEISCHER, A. y MAGEN, N. (2016). «Trust and reputation in the sharing economy: The role of personal photos in Airbnb». Tourism Management, 55, 62-73.

[11] FANG, B.; YE, Q. y LAW, R. (2016). «Effect of sharing economy on tourism industry employment». Annals of Tourism Research, 57, 264-267.

[12] FRADKIN,A.; GREWAL, E.; HOLTZ,D.y PEARSON, M.(2015). «Bias and reciprocity in online reviews: Evidence from field experiments on Airbnb. En Proceedings of the sixteenth ACM conference on economics and computation (EC'15) (pp. 641). New York, NY, EE.UU.: ACM. http://dx.doi.org/10.1145/2764468.2764528.

[13] GUTIÉRREZ, J.; GARCÍA-PALOMARES, J. C.; ROMANILLOS, G. y SALASOLMEDO, M. H. (2017). «The eruption of Airbnb in tourist cities: Comparing spatial patterns of hotels and peer-to-peer accommodation in Barcelona». Tourism Management, 62, 278-291.

[14] GUTTENTAG, D. A. y SMITH, S. L. (2017). «Assessing Airbnb as a disruptive innovation relative to hotels: Substitution and comparative performance expectations». International Journal of Hospitality Management, 64, 1-10.

[15] HAMARI, J.; SJÖKLINT, M. y UKKONEN, A. (2015). «The sharing economy: Why people participate in collaborative consumption». Journal of the Association for Information Science and Technology, 67 (9), 2047-2059.

[16] HEINRICHS, H. (2013). «Sharing economy: a potential new pathway to sustainability». Gaia, 22 (4), 228.

[17] MALHOTRA, A. y VAN ALSTYNE, M. (2014). «The dark side of the sharing economy... and how to lighten it». Communications of the ACM, 57 (11), 24-27.

[18] MARTIN, C. J. (2016). «The sharing economy: A pathway to sustainability or a nightmarish form of neoliberal capitalism?». Ecological Economics, 121, 149-159. 
[19] MOSEDALE, J. (2012). «Diverse economies and alternative economic practices in tourism». En I. Atelvevic, N. Morgan y A. Pritchard (Eds.), The Critical Turn in Tourism Studies: Creating an Academy of Hope (pp. 194-207). Routledge: Abingdon.

[20] PALGAN, Y. V.; ZVOLSKA, L. y MONT, O. (2017). «Sustainability framings of accommodation sharing». Environmental Innovation and Societal Transitions, 23, 7083.

[21] PROGRAMA DE LAS NACIONES UNIDAS PARA EL MEDIO AMBIENTE Y ORGANIZACIÓN MUNDIAL DEL TURISMO (2006). Por un turismo más sostenible. Guía para responsables políticos. Disponible en http://www.unep.fr/shared/publications/pdf/DTIx0884xPA-TourismPolicyES.pdf (visitado el 30 de noviembre de 2017).

[22] SANDOFF, M. (2005). «Customization and standardization in hotels-a paradox or not?». International Journal of Contemporary Hospitality Management, 17 (6), 529535.

[23] SUNDARAJARAN, A. (2014). Peer-to-Peer Businesses and the Sharing (Collaborative) Economy: Overview, Economic Effects and Regulatory Issues. Written testimony for the hearing titled The Power of Connection: Peer to Peer Businesses, January. Disponible en http://smbiz.house.gov/ uploadedfiles/1-15-2014_revised_sundararajan_ testimony.pdf (visitado el 31 de julio de 2017).

[24] TUSSYADIAH, I. P. y PESONEN, J. (2016). «Impacts of peer-to-peer accommodation use on travel patterns». Journal of Travel Research, 55 (8), 1022-1040.

[25] ZERVAS, G.; PROSERPIO, D. y BYERS, J. (2015). «A first look at online reputation on Airbnb, where every stay is above average» (January 28, 2015). SSRN. Disponible en https://ssrn.com/abstract=2554500 (visitado el 31 de julio de 2017).

[26] ZERVAS, G.; PROSERPIO, D. y BYERS, J. W. (2017). «The rise of the sharing economy: Estimating the impact of Airbnb on the hotel industry». Journal of Marketing Research, 54 (5), 687-705. 